\title{
Investigating sepsis with biomarkers
}

In this Practice article (BMJ 2015;350:h254, doi:10.1136/bmj. h254), the first paragraph describing the patient's presentation contains an error. The third sentence should have read: "His urine is cloudy and urinary dipstick test shows the presence of leucocytes and nitrite [not nitrate as was published]."
Cite this as: BMJ 2015;350:h781

๑ BMJ Publishing Group Ltd 2015 